ARTICLE

https://doi.org/10.1038/s41467-019-10665-4

\title{
Local atomic order and hierarchical polar nanoregions in a classical relaxor ferroelectric
}

\author{
M. Eremenko ${ }^{1}$, V. Krayzman ${ }^{1}$, A. Bosak ${ }^{2}$, H.Y. Playford ${ }^{3}$, K.W. Chapman (1) ${ }^{4}$, J.C. Woicik ${ }^{1}$, B. Ravel ${ }^{1}$ \& I. Levin ${ }^{1}$
}

The development of useful structure-function relationships for materials that exhibit correlated nanoscale disorder requires adequately large atomistic models which today are obtained mainly via theoretical simulations. Here, we exploit our recent advances in structure-refinement methodology to demonstrate how such models can be derived directly from simultaneous fitting of 3D diffuse- and total-scattering data, and we use this approach to elucidate the complex nanoscale atomic correlations in the classical relaxor ferroelectric $\mathrm{PbMg}_{1 / 3} \mathrm{Nb}_{2 / 3} \mathrm{O}_{3}$ (PMN). Our results uncover details of ordering of $\mathrm{Mg}$ and $\mathrm{Nb}$ and reveal a hierarchical structure of polar nanoregions associated with the $\mathrm{Pb}$ and $\mathrm{Nb}$ displacements. The magnitudes of these displacements and their alignment vary smoothly across the nanoregion boundaries. No spatial correlations were found between the chemical ordering and the polar nanoregions. This work highlights a broadly applicable nanoscale structurerefinement method and provides insights into the structure of PMN that require rethinking its existing contentious models.

\footnotetext{
${ }^{1}$ Materials Measurement Science Division, National Institute of Standards and Technology, Gaithersburg, MD, USA. ${ }^{2}$ European Synchrotron Radiation Facility, BP 22-, 38043 Grenoble, Cedex, France. ${ }^{3}$ ISIS Facility STFC ISIS Facility, Rutherford Appleton Laboratory, Oxfordshire OX11 0QX, UK. ${ }^{4}$ Department of Chemistry, Stony Brook University, Stony Brook, NY, USA. Correspondence and requests for materials should be addressed to I.L. (email: igor.levin@nist. gov)
} 
A tomic arrangements in most practical materials exhibit some degree of disorder. Glasses or amorphous matter lack structural coherence beyond the first few nearest neighbors, whereas in crystals the disorder exists as a perturbation of an otherwise periodic atomic array. Because of disorder, the local structure-a term that refers to atomic arrangements on a scale ranging from sub-nanometer to several nanometersdiffers from the average. Evidence grows that such fine details of crystal structures, which are largely overlooked by traditional crystallographic methods, control the functional responses of many advanced technological materials. Therefore, the ability to consistently and accurately characterize local structure on the relevant length scales becomes critical for the informed design of materials for applications $s^{1,2}$.

Relaxor ferroelectrics ${ }^{3-5}$ are a classical example of systems with properties controlled by nanoscale atomic order that has remained ill-understood despite over a half century of intense studies using the most advanced measurement and computational techniques. Lead magnesium niobate, $\mathrm{PbMg}_{1 / 3} \mathrm{Nb}_{2 / 3} \mathrm{O}_{3}(\mathrm{PMN})$, and its solid solutions, which exhibit highly attractive piezoelectric characteristics, have been and remain at the center of the quest to understand the structural origins of the relaxor behavior. PMN crystallizes with a perovskite-like structure featuring a nanoscale ordering of $\mathrm{Mg}$ and $\mathrm{Nb}$. The polarizable $\mathrm{Nb}$ and $\mathrm{Pb}$ cations are offset from their respective centrosymmetric positions, thus yielding local electric dipoles. While on average the PMN structure exhibits cubic symmetry (retained down to at least $5 \mathrm{~K}$ ), such polar cation displacements are correlated over a local range; however, the exact nature of these correlations and their relations to the underlying short-range chemical order remain contentious.

Most commonly, the displacement correlations in PMN are perceived to be manifested as polar nanoregions (PNR), several nanometers in size, which emerge below the so-called Burns temperature of $\approx 620 \mathrm{~K}^{6-8}$ and are widely regarded as a key feature associated with the relaxor behavior. A formal definition of PNRs is missing but typically this term refers to finite-size regions, dynamic or static, having nonzero spontaneous electrical polarization. A large fraction of structural research on PMN has involved X-ray and neutron-scattering measurements on single crystals, with a focus on the interpretation of the rich diffusescattering patterns in terms of displacement correlations ${ }^{8-18}$; this diffuse scattering appears below the Burns temperature and becomes increasingly pronounced as temperature is reduced. Various models of PNRs that could give rise to these patterns have been proposed, ranging from static nanodomains having well-defined shapes and boundaries ${ }^{18-20}$ to dynamic displacement patterns controlled by acoustic ${ }^{15}$ and/or optical phonon modes $^{22}$. Some works suggested that the PNRs are distributed in a disordered paraelectric matrix ${ }^{19-21,23}$, whereas recent molecular-dynamics studies ${ }^{17}$ have rejected this interpretation, claiming instead that PNRs are separated by domain walls. Similarly, while some computational studies associate the PNRs with chemically ordered regions $(\mathrm{COR})^{23}$, others question this relation ${ }^{15,22}$. The debate continues in part because no comprehensive structural model of PMN that would provide at least a snapshot of the multiscale correlated disorder, while also accounting for all the available experimental data, has been reported thus far.

Here, we use PMN to demonstrate an approach for obtaining fully atomistic nanoscale structural models directly from the experimental data. We employ large atomic ensembles and a Reverse Monte Carlo (RMC) algorithm to simultaneously fit multiple types of diffraction and spectroscopic data, which notably include three-dimensional (3D) X-ray diffuse-scattering intensity distributions. Our results confirmed the rocksalt-type random-site chemical ordering of $\mathrm{Mg}$ and $\mathrm{Nb}$ and revealed a continuous distribution of the order parameter, with its nanoscale spatial fluctuations yielding regions of stronger order. The displacements of both $\mathrm{Pb}$ and $\mathrm{Nb}$ are aligned preferentially with the $\langle 111\rangle$ directions and occur cooperatively over the nanoscale, forming polar $3 \mathrm{D}$ clusters. The arrangement of these clusters is nonrandom, resulting in aggregate PNRs that incorporate several inequivalent $\langle 111\rangle$ clusters. The local alignment of displacements in such PNRs is maintained over an extended range, but the preferred alignment direction varies within a given region. The displacement magnitudes are largest near the PNR centers and decrease monotonically toward their boundaries, with a smooth transition from one PNR to another. No disordered non-polar matrix could be identified. The magnitudes of the $\mathrm{Pb}$ displacements are enhanced as the local $\mathrm{Mg} / \mathrm{Nb}$ ratio increases; however, no spatial correlations between the PNRs and the much smaller CORs could be observed.

\section{Results}

Reconstructing the nanoscale structure from the diffraction data. We modeled the PMN structure at $300 \mathrm{~K}$ and $200 \mathrm{~K}$ using atomic configurations of $40 \times 40 \times 40$ perovskite unit cells $(320,000$ atoms, $16 \times 16 \times 16 \mathrm{~nm})$, which sample interatomic distances up to $8 \mathrm{~nm}$ (see the Methods section). The atomic coordinates in these configurations were varied according to an RMC algorithm ${ }^{24,25}$ while simultaneously fitting the neutron and $\mathrm{X}$-ray powder total-scattering functions; their respective realspace Fourier transforms, which represent atomic pairdistribution functions (PDF); and 3D distributions of X-ray diffuse intensity measured using a single crystal. In addition, we included the extended X-ray absorption fine structure (EXAFS) data for $\mathrm{Pb}$ and $\mathrm{Nb}$ to provide chemical resolution. The refinements were enabled by several breakthrough developments in the RMC software and methodology which permitted efficient treatment of large atomic configurations, accurate fitting of the total-scattering data to long distances, and fitting of 3D diffuseintensity distributions over an extended range of reciprocal space (see Methods).

A summary of the experimental and fitted signals at $300 \mathrm{~K}$ with satisfactory agreement observed for all the data sets is displayed in Supplementary Fig. 1. Importantly, both neutron and X-ray PDFs are reproduced over the entire distance ranges and the calculated 3D diffuse scattering (Fig. 1; Supplementary Fig. 2) provides a close match to the experimental data. Not only the much-studied
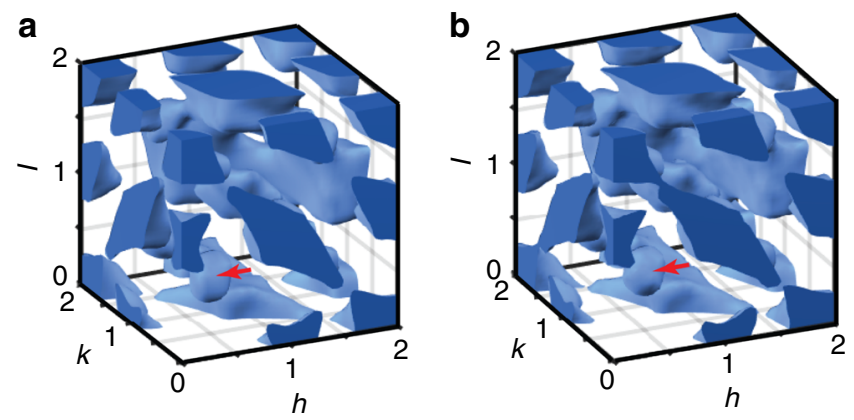

Fig. $1 X$-ray diffuse scattering. a Experimental and $\mathbf{b}$ calculated 3D constantintensity surfaces for the $\mathrm{X}$-ray diffuse-scattering intensity for a $\mathrm{PbMg}_{1}$ / ${ }_{3} \mathrm{Nb}_{2 / 3} \mathrm{O}_{3}$ single crystal at $300 \mathrm{~K}$. The dark shade of blue marks the intersections of these surfaces with the bordering box. Note the distinct shape of the diffuse scattering at the h00-type and hkO-type locations. The 3D diffuse-intensity distribution was fitted simultaneously with the neutron and $\mathrm{X}$-ray total scattering and extended X-ray absorption fine structure data, as summarized in Supplementary Fig. 1. The red arrows indicate the $1 / 2 \quad 1 / 2 \quad 1 / 2$-type peaks associated with the chemical ordering of $\mathrm{Mg}$ and $\mathrm{Nb}$ 
a

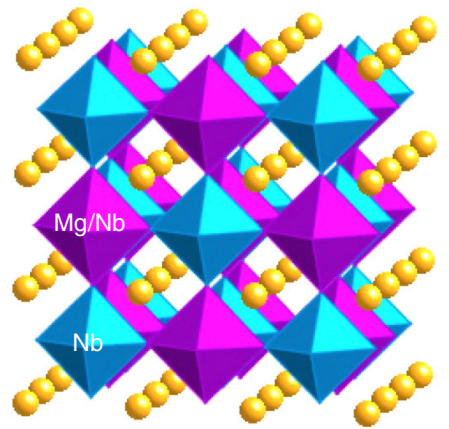

C

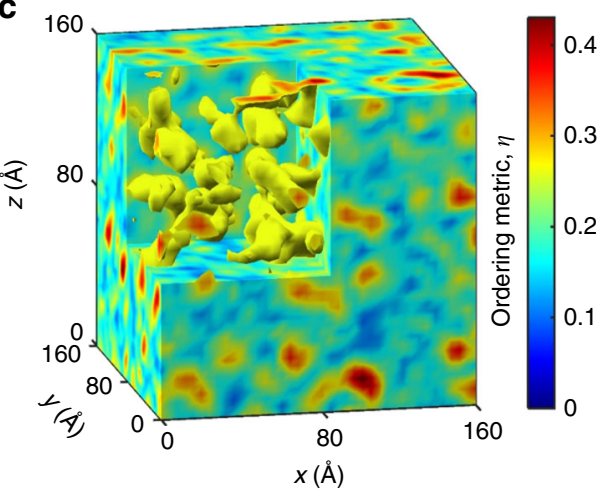

b

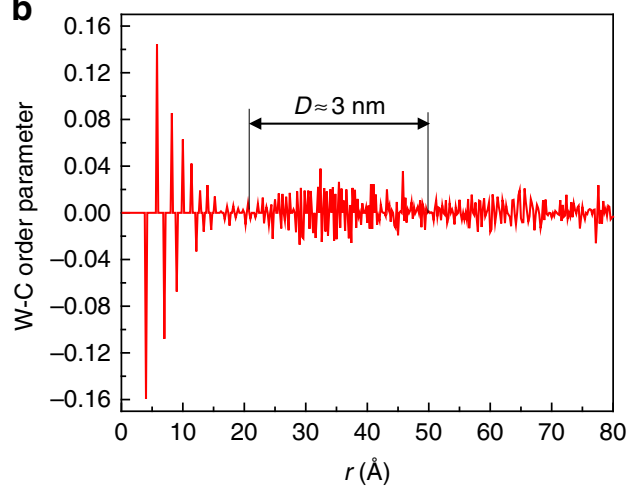

d

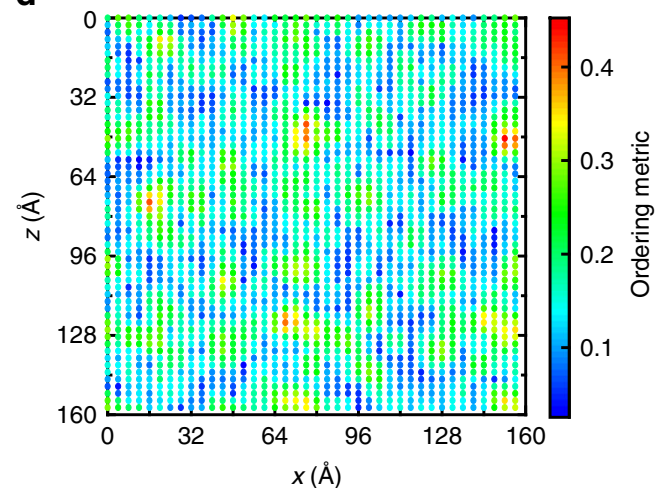

Fig. 2 Characteristics of the chemical ordering of $\mathrm{Mg}$ and $\mathrm{Nb}$. a A schematic rendering of the rocksalt-type partial ordering of $\mathrm{Nb}$ and $\mathrm{Mg}$, with the alternate $\{111\}$ planes occupied by $\mathrm{Nb}$ (blue octahedra) and the $\mathrm{Mg}_{2 / 3} \mathrm{Nb}_{1 / 3}$ (magenta octahedra) mixture, respectively. The Pb atoms are represented using yellow spheres. $\mathbf{b}$ The Warren-Cowley (W-C) short-range order parameter 30 for the distribution of $\mathrm{Mg}$ and $\mathrm{Nb}$, calculated as a function of interatomic distance for the refined configuration. A characteristic length, $D$, that corresponds to the effective size of chemically ordered regions is indicated. $\mathbf{c} A \mathrm{D}$ distribution of the local chemical short-range order parameter, $\eta$ (see the Methods section), which varies continuously across the regions of enhanced order. $\mathbf{d} A$ A map of the local order parameter calculated from the projected Z-map (see text) for the refined configuration as described in ref. 27 . The $x$ and $z$ axes correspond to the [001] and [110] directions, respectively. This map agrees well with its analog derived from the experimental scanning transmission electron microscopy images 26,27

characteristic shapes near the Bragg reflections (i.e., butterfly for $h 00$ and ellipsoidal for $h h 0)$ are reproduced but also the weak diffuse peaks at $1 / 2 h k l(h=2 n+1, k=2 n+1, l=2 n)$ positions (Supplementary Fig. 3) and the overall $Q$-dependence of the diffuse intensity (Supplementary Fig. 4). Similar high-quality fits were obtained for $200 \mathrm{~K}$.

Chemical ordering of $\mathbf{M g}$ and $\mathbf{~} \mathbf{b}$. While the existence of CORs, $2-6 \mathrm{~nm}$ in size, can be regarded as well established, the details of the ordering, which influences the random electric fields and, in turn, the polar displacements ${ }^{26}$, are less certain. Recent results from atomic-resolution imaging in a scanning transmission electron microscope (STEM) ${ }^{27,28}$ and from 3D imaging using Xray resonant scattering ${ }^{29}$ both support a partially ordered $\mathrm{NaCl}$ type 1:1 arrangement with alternate $\{111\}$ planes occupied preferentially by $\mathrm{Nb}$ and a $2: 1 \mathrm{Mg} / \mathrm{Nb}$ mixture, respectively (Fig. 2a). From the STEM images ${ }^{28}$, the CORs appeared to exhibit a smoothly varying order parameter, being separated by wide antiphase boundaries, rather than by a disordered matrix.

In reciprocal space, the cation ordering is manifested in the diffuse peaks at $1 / 2 h k l(h=2 n+1, k=2 n+1, l=2 n+1)$ that are prominent in the single-crystal diffuse-scattering patterns (Fig. 1). We started with a random distribution of $\mathrm{Mg}$ and $\mathrm{Nb}$ on the octahedral sites and fitted these 3D peaks while swapping the locations of the two cations according to the RMC procedure. $\mathrm{No}$ atomic moves other than the $\mathrm{Mg} / \mathrm{Nb}$ swaps were allowed at this stage. The distance dependence of the Warren-Cowley short-range order parameter ${ }^{30}$ calculated for the refined configuration (Fig. 2b) confirms the rocksalt-type ordering and suggests the presence of CORs, $\approx 2-3 \mathrm{~nm}$ in size, without a disordered matrix. The order parameter for the first coordination sphere is at $\approx 70 \%$ of its maximum possible value. Similar results were obtained by starting with a rocksalt-type ordered arrangement of $\mathrm{Nb}$ and $\mathrm{Mg}$ instead of their random distribution.

We used a local short-range ordering metric, $\eta$, calculated similarly as described in ref. ${ }^{28}$, to visualize a spatial distribution of the ordered regions. A 3D map of this metric for the refined configuration (Fig. 2c) reveals the CORs, which appear as the quenched spatial fluctuations of the order parameter, while the degree of ordering varied continuously across the system. We compared our results with those inferred from the experimental STEM images by calculating a (110) projection of our configuration box, with the projected atomic columns assigned intensities according to their average atomic numbers $(Z)$ squared. The resulting image, which we call a $Z$-map, approximates the $Z$ contrast in STEM high-angle annular dark-field images (Supplementary Fig. 5a). The Fast Fourier transform (FFT) of this $Z$-map yields the $1 / 21 / 21 / 2$-type superlattice diffuse spots like those obtained experimentally. Comparison of the calculated map and the experimental image, both FFT-filtered using these spots, demonstrates their qualitative agreement (Supplementary Fig. 5). Moreover, a map of the 2D ordering metric (Fig. 2d), calculated exactly as suggested in ref. ${ }^{28}$, closely matches a similar map obtained by these authors from the experimental images 
a

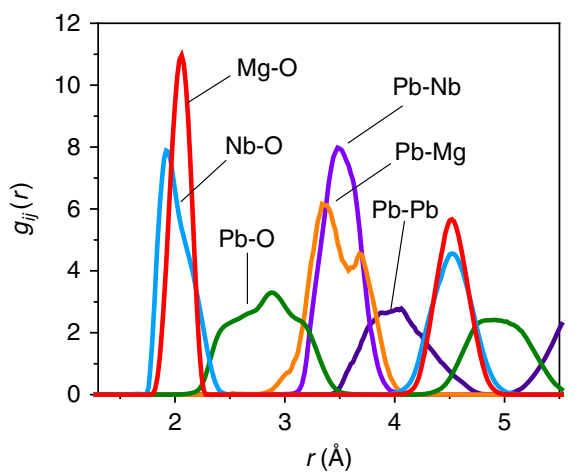

c

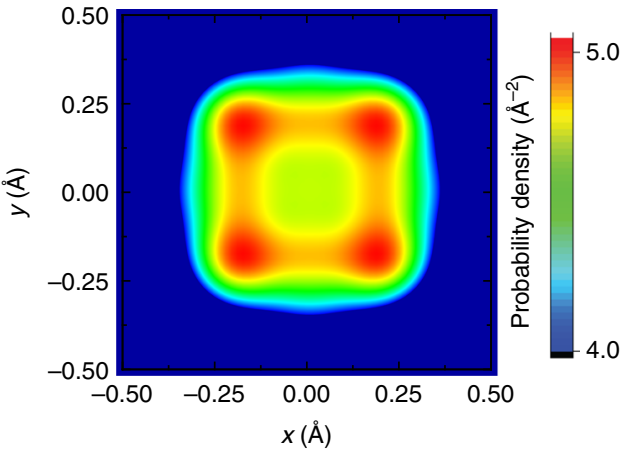

b

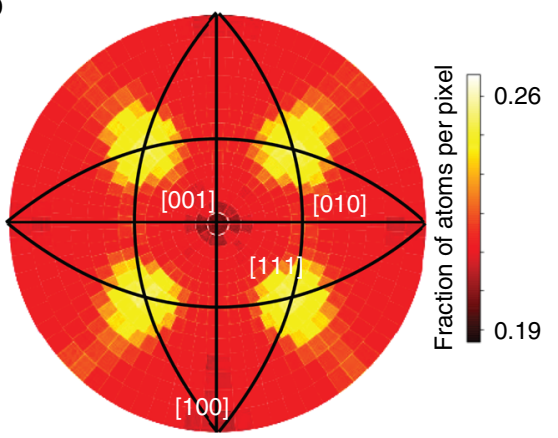

d

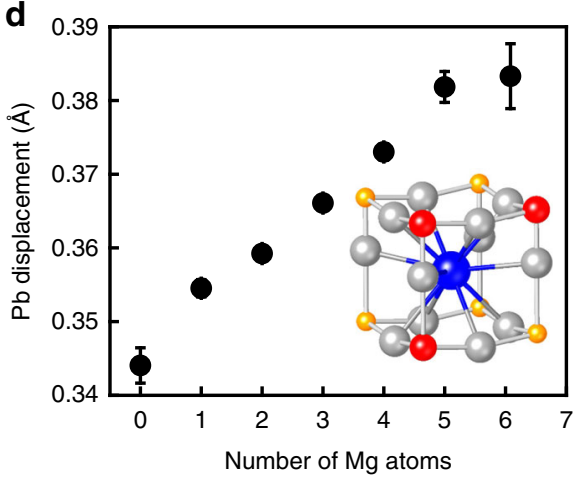

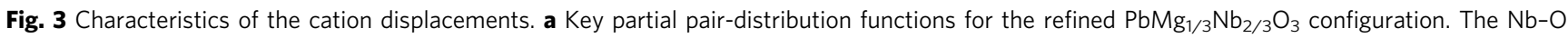
and $\mathrm{Pb}-\mathrm{O}$ peaks are split, suggesting off-centering of the respective cations. In contrast, the $\mathrm{Mg}-\mathrm{O}$ distribution exhibits a single symmetric peak. b A stereographic-projection map of the Pb displacements demonstrating their preference for the $\langle 111\rangle$ directions. (The black lines indicate traces of

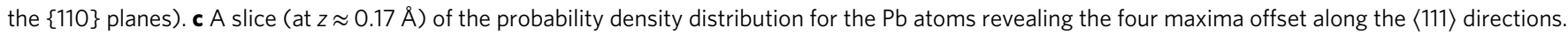
d Magnitude of the $\mathrm{Pb}$ displacements as a function of the local $\mathrm{Mg} / \mathrm{Nb}$ ratio around $\mathrm{Pb}$. The inset illustrates the $\left[\mathrm{PbO}{ }_{12}(\mathrm{Mg} / \mathrm{Nb})_{8}\right] \mathrm{coordination}$. $\mathrm{Pb}, \mathrm{O}, \mathrm{Mg}$, and $\mathrm{Nb}$ species are represented by the blue, light gray, red, and yellow spheres, respectively. The error bars represent single standard deviations estimated by analyzing three independently refined configurations

(the thickness of their TEM samples was comparable with the dimension of our configuration box along the projection direction). Therefore, we concluded that the presently obtained models accurately represent the state of ordering in PMN and have adopted them for further refinements of atomic displacements (performed without any swap moves); the same $\mathrm{Mg} / \mathrm{Nb}$ distribution was used for both $300 \mathrm{~K}$ and $200 \mathrm{~K}$ since the metalion diffusion at these temperatures is negligible.

Cation displacements and PNRs. Partial metal-oxygen PDFs (Fig. 3a) calculated from the refined atomic coordinates confirm that both $\mathrm{Pb}$ and $\mathrm{Nb}$ are off-centered within the respective oxygen coordination polyhedra (cuboctahedra for $\mathrm{Pb}$ and octahedra for $\mathrm{Nb}$ ), while $\mathrm{Mg}$ remains approximately central. The double-peak $\mathrm{Pb}-\mathrm{Mg}$ and single-peak $\mathrm{Pb}-\mathrm{Nb}$ distributions indicate much stronger off-centering of $\mathrm{Pb}$ relative to its $\mathrm{Mg}$ neighbors as compared with $\mathrm{Nb}$, which is in line with the recent inferences from quantitative STEM imaging 27 . On average, all the cations retain their ideal cubic positions. Locally, however, both $\mathrm{Pb}$ and $\mathrm{Nb}$ atoms are displaced preferentially along the $\langle 111\rangle$ directions, and this preference becomes more pronounced on cooling from $300 \mathrm{~K}$ to $200 \mathrm{~K}$ (Fig. 3b; Supplementary Fig. 6); the displacements of $\mathrm{Mg}$ appear to be isotropic. The probability density distribution (PDD) of $\mathrm{Pb}$ features eight well-separated $\langle 111\rangle$ maxima (Fig. $3 \mathrm{c}$ ) with the $\mathrm{Pb}$ atoms offset by $\approx 0.3 \AA$ from the ideal cubic position. In contrast, the PDDs of $\mathrm{Nb}$ and $\mathrm{Mg}$ appear as a single peak. The $\mathrm{Nb}$ PDD exhibits a flattened top, which suggests either a flat potential well or an unresolved Nb-site splitting, whereas the $\mathrm{Mg}$ distribution is Gaussian. The root-mean-square displacements of $\mathrm{Nb}$ and $\mathrm{Mg}$ are $\approx 0.19 \AA$ and $\approx 0.11 \AA$, respectively.

The observed off-center displacements of $\mathrm{Pb}^{2+}$ and $\mathrm{Nb}^{5+}$ agree with the known tendencies of these cations to form short, strongly covalent bonds with oxygen via hybridization of their respective $6 \mathrm{~s}$ and $4 \mathrm{~d}$ states with $\mathrm{O} 2 \mathrm{p}$ states. The magnitudes of the local $\mathrm{Pb}$ displacements increase with the $\mathrm{Mg} / \mathrm{Nb}$ ratio in the $\left[\mathrm{PbNb}_{8-n} \mathrm{Mg}_{n}\right]$ clusters (Fig. 3d), with a similar dependence obtained for both $300 \mathrm{~K}$ and $200 \mathrm{~K}$. This trend can be attributed to the lower ionic charge of $\mathrm{Mg}$ and hence its less covalent bond with oxygen, which results in increasingly underbonded-oxygen atoms as $n$ increases; therefore, larger $\mathrm{Pb}$ shifts are required to satisfy the oxygen-bonding requirements. Concurrently, $\mathrm{Mg}^{2+}$ has a larger ionic radius $(\approx 0.70 \AA)$ than $\mathrm{Nb}^{5+}(\approx 0.64 \AA)^{31}$ resulting in the larger size of the cuboctahedral cages for the Mg-rich configurations; this ionic-radii difference is comparable to the difference in the values of $\mathrm{Pb}$ displacements for $n=0$ and $n=7$ (Fig. 3d).

The underbonded-oxygen argument also applies to the effects of $\mathrm{Pb}$ off-centering on the ordering of $\mathrm{Mg}$ and $\mathrm{Nb}$. This offcentering, which modifies the bonding state of oxygen, has been previously suggested to stabilize the $\mathrm{Mg} / \mathrm{Nb}$ configurations that could be electrostatically less favorable ${ }^{32}$. In fact, competition between long-range electrostatic and short-range $\mathrm{Pb}-\mathrm{O}$ interactions could be a reason for favoring a partially ordered 1:1 type array of $\mathrm{Mg}$ and $\mathrm{Nb}$ over the completely ordered 1:2 layered arrangement. Thus, the existence of a relationship between the degree of $\mathrm{Mg} / \mathrm{Nb}$ ordering and at least the static $\mathrm{Pb}$ displacements is chemically reasonable. We do observe that the overall $\mathrm{Pb}$ 
a

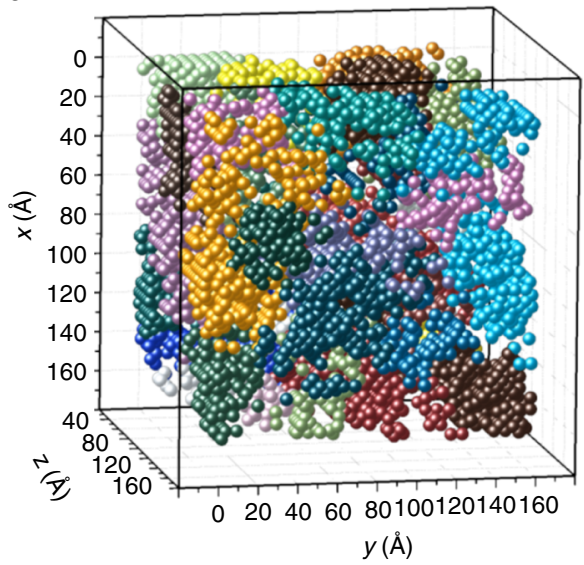

b

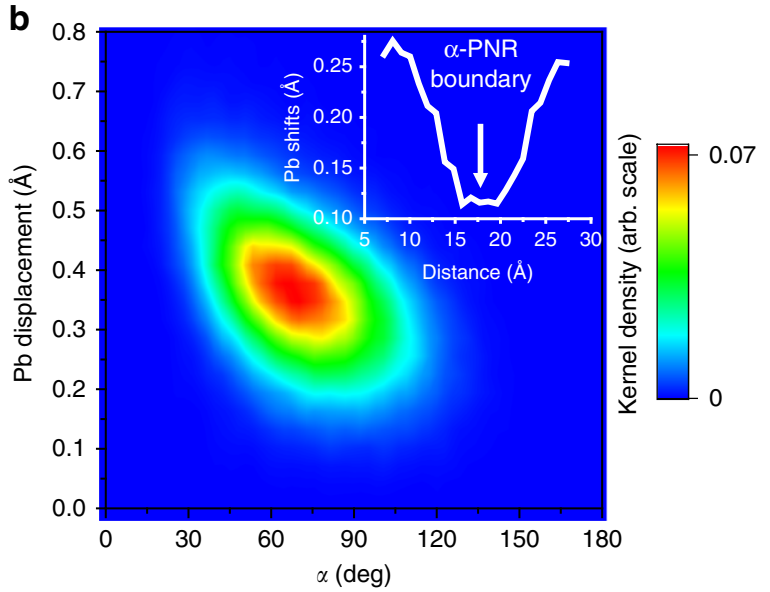

d
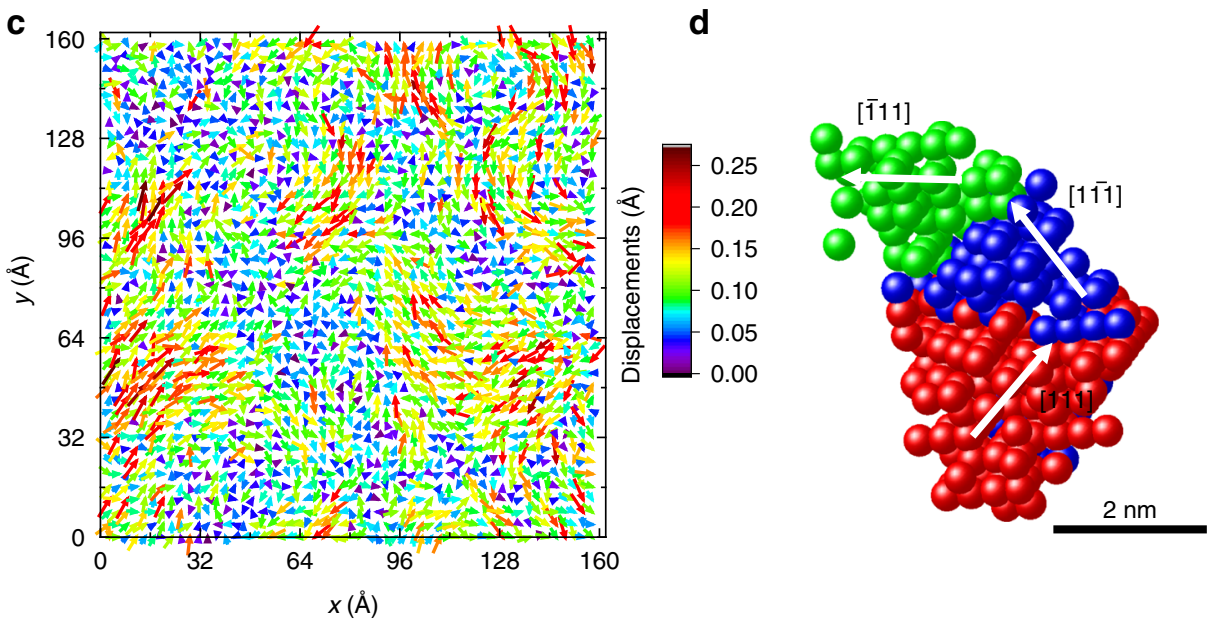

Fig. 4 Correlations among the $\mathrm{Pb}$ displacements. a Clusters of $\mathrm{Pb}$ atoms having a local displacement-alignment metric $\alpha$ (see text) for the first $\mathrm{Pb}$ - $\mathrm{Pb}$ coordination sphere less than $45^{\circ}$ (spatially distinct clusters are labeled using different colors). As a reference, $\alpha=0^{\circ}$ and $\alpha=180^{\circ}$ correspond to the parallel and antiparallel displacements of a given atom and its neighbors, respectively. The clusters have been identified according to the HDBSCAN 33 algorithm. The search procedure accounted for the periodic boundary conditions; however, for visualization purposes, we assigned a distinct color to each region within the configuration box. b Magnitudes of the $\mathrm{Pb}$ displacements plotted against $\alpha$; a strong negative correlation between the two characteristics is observed. The inset illustrates variation of the displacement magnitude for the Pb columns across the $\alpha$-PNR boundary, as derived from panel $\mathbf{c}$. c A 2D map of displacements for the $\mathrm{Pb}$ columns projected onto a $\{110\}$ plane; the color-scale bar reflects the displacement magnitude in $\AA$ and the arrows are used to illustrate the displacement directions. The $x$ and $y$ axes correspond to the orthogonal [110] and [001] directions. This distribution agrees with the analogous maps deduced from experimental atomic-resolution scanning transmission electron microscopy images of $\mathrm{PbMg}_{1 / 3} \mathrm{Nb}_{2 / 3} \mathrm{O}_{3}$. The displacements decay monotonically toward the boundaries. d 3D rendering of a representative $\alpha$-PNR, with the constituent Pb atoms (represented by spheres) that are displaced along the inequivalent $\langle 111\rangle$ directions (labeled in the figure) indicated using different colors

displacements in the instantaneous configuration are enhanced in the cores of CORs if only slightly; the small magnitude of this effect is determined by the continuously varying degree of ordering (see Supplementary Fig. 7 for a detailed analysis).

The $3 \mathrm{D} \mathrm{Pb}$-displacement correlations in the form of dense nanoscale regions are evident from considering the local alignment metric, $\alpha$, defined as the average of the angles between the displacement vectors of a given atom and each of its neighbors within a sphere of a certain radius. Figure 4 a displays the $\mathrm{Pb}$ atoms with $\alpha<45^{\circ}$ (for the first $\mathrm{Pb}-\mathrm{Pb}$ coordination shell) that have been identified by the HDBSCAN algorithm ${ }^{33}$ to form dense, spatially distinct clusters (labeled using different colors). In this representation, PMN appears as an assemblage of regions, about $4-6 \mathrm{~nm}$ in size, featuring the locally aligned displacements; we will call these regions $\alpha$-PNRs. The displacements of $\mathrm{Nb}$ are strongly and positively correlated with those of $\mathrm{Pb}$ (Supplementary Fig. 8). Presumably, these correlations are driven by the bonding requirements of the oxygen atoms.
The values of $\alpha$ are minimal near the centers of $\alpha$-PNRs and increase gradually toward their boundaries. The magnitude of the $\mathrm{Pb}$ displacements scales inversely with $\alpha$ (Fig. 4b). Overall this picture agrees with the results of the recent MD studies ${ }^{17}$, which suggested a high incidence of domain walls without a disordered matrix. Projections of the $\mathrm{Pb}$-displacement field onto the $\{110\}$ planes also agree with the displacement patterns identified in the recently published STEM images ${ }^{27}$ (Fig. 4c). Figure $4 \mathrm{~b}$ (inset) illustrates smooth variation of the displacement magnitude across the $\alpha$-PNR boundary. Our refinements at $200 \mathrm{~K}$, which is below the freezing temperature $T_{\mathrm{f}} \approx 230 \mathrm{~K}$, reveal significant growth of the $\alpha$-PNRs with a concurrent increase in the magnitudes of the $\mathrm{Pb}$ displacements (Supplementary Fig. 9). This observation agrees with the previously reported experimental and modeling data on the temperature behavior of PMN. Also, the estimated sizes of $\alpha$ PNRs are comparable with the correlation lengths identified in earlier studies using parameterized fits of variable-temperature neutron PDFs'. 
The definition of PNRs according to the local alignment angle disregards displacement directions. We added this directional specificity by identifying those $\mathrm{Pb}$ atoms that have their displacement vectors aligned with the $\langle 111\rangle$ directions. $\mathrm{Pb}$ atoms displaced along a given $\langle 111\rangle$ direction also form extended $3 \mathrm{D}$ clusters, which we will refer to as $\langle 111\rangle$-PNRs. The $\langle 111\rangle$ PNRs and $\alpha$-PNRs have comparable sizes, however, the correlation length of the displacement magnitudes within the $\langle 111\rangle$-PNRs is considerably shorter than that of the displacement directions.

Typical $\alpha$-PNRs are composed of portions of $2-3\langle 111\rangle$-PNRs, which are arranged so that the adjacent regions are preferentially $71^{\circ}\langle 111\rangle$ variants (Fig. 4d; Supplementary Fig. 10). This preference, which also agrees with the MD simulations ${ }^{17}$, is evident both from the analysis of the individual $\alpha$-PNRs and from the histograms that describe distributions of distances between the $\mathrm{Pb}$ atoms that belong to the $71^{\circ}, 109^{\circ}$, and $180^{\circ}$ variants, respectively (Supplementary Fig. 11). Thus, PMN exhibits a hierarchical PNR structure, with the correlated arrangement of the $\langle 111\rangle$-PNRs yielding the aggregate $\alpha$-PNRs; this aggregate nature of $\alpha$-PNRs is retained even if the threshold value of $\alpha$ used to identify them is reduced. No relation could be found between the PNRs and the significantly smaller CORs; the size difference between these two types of regions becomes particularly pronounced at $200 \mathrm{~K}$.

Diffuse-scattering simulations using only those $\mathrm{Pb}$ atoms that belong to a single variant (out of 8 ) of the $\langle 111\rangle$-PNRs could not reproduce the characteristic shapes of the diffuse scattering, which emerged only in the presence of all the variants. This result resonates with the assessment by Takenaka et al. ${ }^{17}$, who attributed the diffuse scattering in PMN to a multidomain state. We investigated the nature of such a multi-domain state by performing an inverse Fourier transform (IFT) of the calculated diffuse-scattering amplitude around the Bragg peaks (spherical masks $0.2 \AA^{-1}$ in diameter were used). Extensive testing using simulated structures demonstrated that IFT successfully recovers regions of correlated displacements and the displacement patterns that underlie specific diffuse-scattering features. The $\mathrm{Pb}$ atoms that are associated with the largest variation of the amplitude of the IFT (see Methods) form 3D clusters each composed predominantly of the two $71^{\circ}\langle 111\rangle$-PNRs with nearly all the $\mathrm{Pb}$ atoms having $\alpha<70^{\circ}$ (Supplementary Fig. 12); a spatial distribution of these regions resembles that of $\alpha$-PNRs. For an aggregate of the two equal-volume $71^{\circ}\langle 111\rangle$-PNRs, the effective polarization direction is $\langle 110\rangle$, which is consistent with the anisotropy of the diffuse-scattering distributions. Thus, the much-studied diffuse-scattering features appear to arise from $\alpha$ PNRs, which develop because of the correlated spatial arrangements of $\langle 111\rangle$-PNRs coupled with concurrent modulation of the displacement-magnitudes.

We also applied IFT to the calculated diffuse-scattering peaks at the $1 / 21 / 20$-type locations (Supplementary Fig. 3), the so-called M-points, which previously have been attributed to local ordering of antiparallel cation displacements that signify antiferroelectric interactions ${ }^{18,34}$. The analysis of these weak peaks is complicated because they reside on a large background and overlap with the diffuse-intensity tails that extend from the Bragg reflections (ref. ${ }^{34}$; Supplementary Fig. 13). Nevertheless, by zooming in on the locations of the $\mathrm{Pb}$ atoms that exhibit the largest variation of the IFT amplitude associated with the M-points, we could identify the nanoscale antiparallel ordering of the $\langle 100\rangle \mathrm{Pb}$-displacement components (depicted schematically in Supplementary Fig. 14). This ordering differs from the model with antiparallel $\langle 110\rangle \mathrm{Pb}$ displacements proposed in ref. ${ }^{34}$; however, we could not discriminate the background effects sufficiently to visualize the entire population of such ordered clusters responsible for the M- points and, therefore, the presence of several types of displacement patterns cannot be ruled out.

Coupling of cation displacements to the oxygen framework. Concerted cation displacements in the PNRs are expected to be accompanied by characteristic distortions of oxygen octahedra. We analyzed local distortions of the octahedral framework by expanding oxygen displacements for the individual octahedra into a set of deformation modes, and then searched for correlations among these modes and the cation displacements. A full orthonormal basis of the 18 deformation modes for an octahedron (Supplementary Fig. 15) was defined to reflect common lattice distortions (three tetragonal, three orthorhombic, and three octahedral rotations), with the remaining modes (three translational, six bending, $\mathrm{F}_{1 \mathrm{u}^{-}}$and $\mathrm{F}_{2 \mathrm{u}}$ ) selected to provide a complete set. In this basis, a symmetric octahedron's breathing mode $\left(A_{1 g}\right)$ is the sum of the three tetragonal modes, whereas the rhombohedral deformations can be calculated from the combinations of the orthorhombic and tetragonal modes. The amplitudes of the 18 basis modes were determined for every octahedron in the configuration.

Only the translational and the bending $\mathrm{F}_{1 \mathrm{u}}$ (Supplementary Fig. 15) modes appeared to be coupled to the directions of the cation displacements. The $\mathrm{Pb}$ displacements are negatively correlated with those of oxygen in these modes for both the $\left[\mathrm{Pb}_{8}\left(\mathrm{NbO}_{6}\right)\right]$ and $\left[\mathrm{Pb}_{8}\left(\mathrm{MgO}_{6}\right)\right]$ clusters. Similar negative correlations are observed for the $\mathrm{Nb}$ displacements, whereas for $\mathrm{Mg}$ the correlations are strongly positive. The resulting oxygen-cation displacement patterns (Supplementary Fig. 16) match the lowfrequency transverse optical modes commonly encountered in $\mathrm{PMN}^{35}$ and other perovskite ferroelectrics, which indicates that even such complex multiatomic correlations are reproduced in the refined configurations. Similar trends hold for the $x, y$, and $z$ axis components of the deformation modes and cation displacements. No dependence of the deformation-mode amplitudes and the cation-oxygen coupling on the chemical order parameter was observed. As a soundness check for the procedure, the mean amplitudes of the breathing modes for the $\left[\mathrm{NbO}_{6}\right]$ and $\left[\mathrm{MgO}_{6}\right]$ octahedra were found to be negative and positive, respectively, which reflects oxygen displacements away from $\mathrm{Mg}$ toward $\mathrm{Nb}$, consistent with the $\mathrm{Mg}^{2+}-\mathrm{Nb}^{5+}$ ionic charge difference and the stronger covalency of the $\mathrm{Nb}-\mathrm{O}$ bonds; the values of the two amplitudes are correctly related according to the $\mathrm{Nb} / \mathrm{Mg}$ stoichiometry. The observed correlated oxygen displacements account for a distinct appearance of the neutron single-crystal diffuse scattering near the $h 00$ Bragg peaks with $h=2 n$ and $h=2 n+1$ (Fig. 5); the agreement with the experimental plots reported in ref. ${ }^{18}$ is especially striking given that these data were not included in the present fits.

Atomic configurations obtained from X-ray and neutronscattering data represent structural snapshots with superposition of both the static and dynamic displacements (see the Methods section for a discussion of the effects of energy integration on both types of data); the latter reflect contributions from acoustic and low-frequency optical phonons. Our observations of the negative correlations between the oxygen and $\mathrm{Pb} / \mathrm{Nb}$ displacements indicate that the oxygen-shifts involved in such optical modes are sufficiently large to overcome the usually dominant effect of acoustic motion.

\section{Discussion}

The combination of X-ray and neutron total-scattering data with a $3 \mathrm{D}$ distribution of diffuse-scattering intensity has proven to be effective for unraveling complex nanoscale perturbations of the average structure, such as encountered in PMN; our tests 


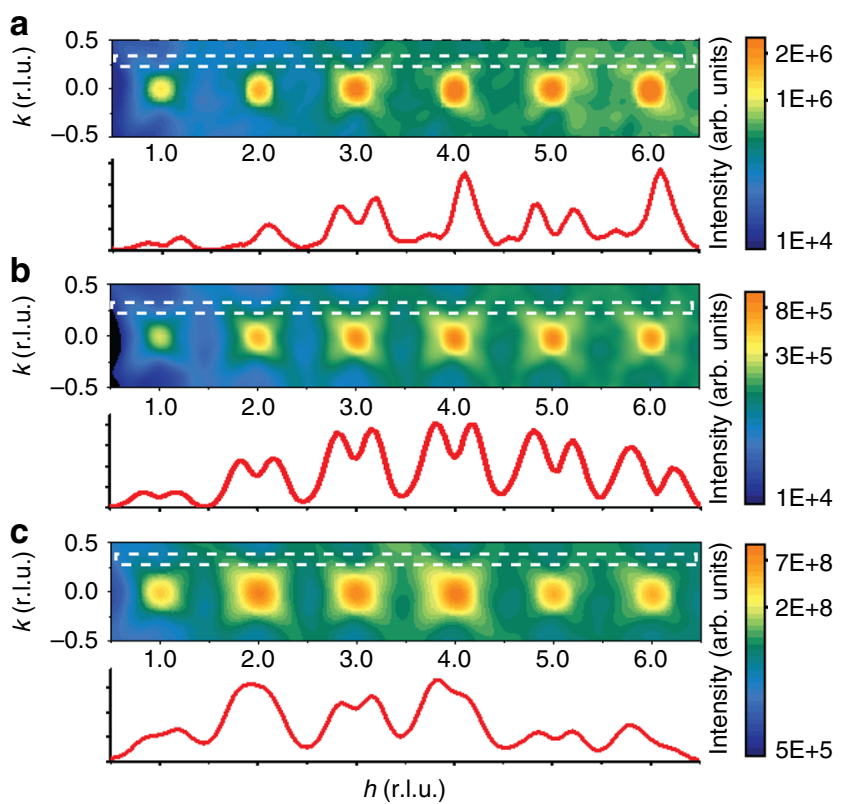

Fig. 5 Simulated neutron diffuse scattering. a, b Neutron diffuse scattering near the $h 00$ reflections ( $h$ is the horizontal axis) calculated for the refined configuration at $300 \mathrm{~K}$ with (a) all the species and (b) only the $\mathrm{Pb}$ atoms included in the calculations. $\mathbf{c} X$-ray diffuse scattering near the same reflections with all the species included in the calculations. The intensity traces correspond to the $k$-positions indicated using white/dotted rectangles; the logarithmic intensity scales are the same as indicated in the corresponding color-scale bars. The neutron scattering in (a) is modulated relative to (b), featuring different appearance for the asymmetry of the $h 00$ reflections with $h=2 n$ and $h=2 n+1$. This modulation, which is associated with the correlated oxygen displacements, reproduces the experimental results reported in ref. ${ }^{18}$, even though the present refinements did not include neutron diffuse scattering

demonstrated that a complete picture could not be recovered using any of these techniques alone. The reconstructed chemical and displacive nanoscale ordering in PMN agrees well with the reported STEM images ${ }^{27,28}$, which attests to the fidelity of the present structural models and of the information that can be extracted from quantitative STEM measurements. The obtained atomistic picture invalidates the notion of chemical or polarordered regions dispersed in a disordered matrix, instead suggesting continuously varying order parameters. The displacements of $\mathrm{Pb}$ and $\mathrm{Nb}$ atoms at both $300 \mathrm{~K}$ and $200 \mathrm{~K}$ exhibit preference for the $\langle 111\rangle$ directions, in contrast to previously proposed spherical-shell distributions. The $\langle 111\rangle$ displacements are spatially correlated, but an additional higher-level correlation exists that is manifested in a preference for neighboring $\langle 111\rangle$ clusters to represent $71^{\circ}$ variants, resulting in a hierarchical assembly of PNRs; displacement-magnitudes follow this latter correlation. Preference for the $71^{\circ}$ variants has also been noted in the most recent $\mathrm{MD}$ simulations of $\mathrm{PMN}^{17}$, which together with our results point to the significance of this effect. Consistent with the previously made inferences, the PNRs grow on cooling to $200 \mathrm{~K}$. In addition, nanoscale regions with ordered antiparallel $\mathrm{Pb}$ displacements are shown to exist alongside the PNRs. While we observe no spatial coincidence between the PNRs and CORs, chemical ordering is still expected to affect the behavior of polar displacements, as can be inferred from the dependence of $\mathrm{Pb}$ displacements on the local $\mathrm{Mg} / \mathrm{Nb}$ ratio. Our results suggest that the existing models of PMN that ignore the continuously varying degree of chemical ordering and overlook the inter-PNR correlations should be reconsidered. Understanding the origins of the
inter-PNR correlation, its evolution with temperature and chemical substitutions, and ultimately its significance for the dielectric response represent topics for future theoretical and experimental research on PMN and its solid solutions. A logical step would be to perform similar refinements but add the $3 \mathrm{D}$ neutron diffuse scattering ${ }^{18}$ to the data suite, which should provide more accurate correlations of the oxygen displacements.

As a more general remark, we note that with recent advances in neutron and synchrotron instrumentation, measurements of 3D diffuse-intensity distributions over a broad range of reciprocal space have been facilitated, creating opportunities for wider use of the approach described in this study. The accuracy and scope of structural information that can be recovered using combinedtechnique refinements are largely limited by the quality of experimental data and the sizes of atomic configurations that can be handled within reasonable computing times. The currently used atomic configurations are still at the lower limit of what is required for accurate treatment of nanoscale correlations in systems like PMN. In fact, since the PNRs grow at $200 \mathrm{~K}$, this configuration-size already becomes insufficient. Thus, harnessing the full power of nanoscale atomistic structural refinements requires improved instrumentation, more accurate datareduction procedures, and efficient structure-refinement computer algorithms that would take full advantage of the underused computing resources available today.

\section{Methods}

Synthesis. A pyrochlore-free ceramic sample of PMN was prepared by solid-state reaction according to the columbite route proposed in ref. ${ }^{35}$. Powders of $\mathrm{MgO}$ and $\mathrm{Nb}_{2} \mathrm{O}_{5}$ (with $2 \mathrm{~mol} \%$ excess $\mathrm{MgO}$ ) were reacted at $1000^{\circ} \mathrm{C}$ for $8 \mathrm{~h}$ to form $\mathrm{MgNb}_{2} \mathrm{O}_{6}$. This product was batched with a stoichiometric amount of $\mathrm{PbO}$ and heated in a covered crucible at $800^{\circ} \mathrm{C}$ for $4 \mathrm{~h}$ to form phase-pure PMN. Prior to each heating, the powders were mixed and ground in a planetary ball mill in isopropanol using yttria-stabilized zirconia grinding media.

Total-scattering measurements. Neutron total-scattering data were collected at $300 \mathrm{~K}$ and $200 \mathrm{~K}$ using the Polaris diffractometer at the ISIS facility (Science and Technology Facility Council, UK). The sample powder was loaded in a vanadium can with temperature control achieved using a displex cryostat. The data were processed using the GUDRUN software to obtain the total-scattering function $S(Q)$ and its Fourier transform, $G(r)\left(Q_{\max }=35 \AA^{-1}\right)$. X-ray total scattering was measured at the 11-ID-B beamline of the Advanced Photon Source (Argonne National Laboratory) using an incident-beam energy of $\approx 60 \mathrm{keV}$ with the area detector positioned at $18 \mathrm{~cm}$ downstream from the sample. The sample was loaded in a 1 mm kapton capillary. The X-ray data were processed using the PDFGetX3 software to obtain the corresponding total-scattering function and its Fourier transform $\left(Q_{\max }=25 \AA^{-1}\right)$. In both neutron and X-ray total-scattering measurements, the instrument resolution was characterized by measuring NIST Si SRM powder.

Extended X-ray absorption fine structure (EXAFS) measurements were performed for the $\mathrm{Pb} L_{3}$ and $\mathrm{Nb} K$ edges at the NIST beamline 06-BM at NSLS-II (Brookhaven National Laboratory). The data were collected at room temperature in transmission. All the data were processed using Athena ${ }^{36}$. Initial fitting was accomplished in the Artemis software ${ }^{37}$. Scattering amplitudes and phases were calculated using FEFF8 ${ }^{38}$. For the Nb EXAFS, the $k$-space data were multiplied by $k^{2}$ prior to the Fourier transform, which was performed over the $k$-range from $3.3 \AA^{-1}$ to $13.2 \AA^{-1}$; the $r$-space fitting range was from $1 \AA$ to $4.2 \AA$, with both single- and multiple-scattering paths of a photoelectron included in the fit. For $\mathrm{Pb}$ the $k$-space data were multiplied by $k$ and the $k$-space range used in the transform extended from $2.2 \AA^{-1}$ to $10 \AA^{-1}$; the $r$-space fit was performed from $1 \AA$ to $3.5 \AA$, with only the single-scattering paths included.

Transmission electron microscopy. Transmission electron microscopy (TEM) was performed on the crushed powder dispersed on a lacey carbon-coated copper grid. Selected area electron diffraction patterns and high-angle annular dark-field images were collected at $300 \mathrm{kV}$ in the TEM and scanning TEM modes, respectively.

Single-crystal X-ray diffuse scattering. X-ray single-crystal scattering measurements were performed at the beamline ID29 of ESRF. A rod-like $50-\mu$ m-thick crystal was mounted on a rotation stage and held in a cryostream flux for tem perature control. Prior to the measurements, the sample was etched using hot concentrated hydrochloric acid. Diffuse-scattering patterns were recorded with the wavelength of $0.954 \AA$ over the angular range of $360^{\circ}$ in $0.1^{\circ}$ increments on a 
single-photon counting pixel detector, which allowed for the shutter-less data collection. The CrysAlis software package was used to refine the orientation matrix and the final reciprocal-space reconstructions of 3D diffuse-intensity distributions were accomplished with locally developed computer software.

Structure refinements. Structural refinements were performed using the development version of the RMCProfile software ${ }^{24}$. This version makes extensive use of GPU computing (yet unavailable for the distribution package of RMCProfile), which is critical for achieving computational speeds required to handle large atomic configurations and calculations of 3D diffuse scattering involved in this study. The PMN structure was modeled using atomic configurations of $40 \times 40 \times$ 40 unit cells, which included 320,000 atoms $(\mathrm{Mg} / \mathrm{Nb}$ ratio is equal to 0.49998828$)$ and covered interatomic distances up to $8 \mathrm{~nm}$. Neutron- and X-ray total-scattering data in both real and reciprocal spaces were fitted simultaneously with the two EXAFS data sets and the 3D X-ray single-crystal diffuse-intensity distribution; in addition, the neutron Bragg profile was included in the fit. The X-ray data were calculated considering the Q-dependence of the X-ray scattering cross-sections.

In both the neutron and X-ray cases, the calculated total-scattering data were corrected for the instrument resolution in reciprocal and real spaces prior to comparison with the corresponding experimental signals. The resolution corrections accounted for the actual peak profile shapes, which is especially important for the time-of-flight neutron data that exhibit significant peak asymmetry. The accuracy of these corrections is key for obtaining meaningful results while fitting a pair-distribution function to longer distances. The 3D X-ray diffuse scattering has been calculated according to the formalism outline by Butler and Welberry ${ }^{39}$. Prior to comparison with the experimental data, the calculated diffuse-scattering intensity was smoothed using a 3D Gaussian filter. The weights assigned to individual data sets, which control the contribution of a given data set to the total residual, were adjusted during fitting using a newly developed automated routine that ensures that all individual residual terms, while allowed to increase temporarily, over time decrease to small user-preset values. This procedure, which involves an analysis of statistical correlations between changes in the aggregate residual function and each of its terms, drastically accelerates convergence of RMC refinements.

For X-ray total scattering, the energy-integration range is always significantly broader than a span of phonon energies and therefore these data reflect instantaneous atomic positions. For EXAFS, a characteristic X-rays-sample interaction time is $\approx 10^{-15} \mathrm{~s}$ thus also probing an instantaneous PDF. In the case of neutron total scattering, the actual energy-integration range is limited by the socalled Placzek shift and reportedly can be less than $10 \mathrm{meV}^{40,41}$. However, for a given material, the exact value of this limit will vary between different instruments and is somewhat uncertain ${ }^{40}$. According to previous studies of PMN using a dynamic $\mathrm{PDF}^{41}$, at $T \geq 450 \mathrm{~K}$, the off-centering of $\mathrm{Pb}$, which is manifested in the splitting of the nearest-neighbor $\mathrm{Pb}-\mathrm{O}$ peak with the appearance of short $\mathrm{Pb}-\mathrm{O}$ distances at $\approx 2.4 \AA$, is purely dynamic. Our variable-temperature X-ray PDF (XPDF) data (Supplementary Fig. 17a) demonstrate that this splitting and the short $\mathrm{Pb}-\mathrm{O}$ distances are observable at least up to $490 \mathrm{~K}$, as expected since X-ray total scattering reveals both static and dynamic correlations. Our neutron PDF (N-PDF) at $490 \mathrm{~K}$ (Supplementary Fig. 17b) also features a clearly identifiable splitting of the $\mathrm{Pb}-\mathrm{O}$ distribution with a peak at $\approx 2.4 \AA$, as confirmed by the presence of anomalies in the second derivative of the PDF signal (Supplementary Fig. 17c). Considering that the onset energy for a phonon that involves the off-centering of $\mathrm{Pb}$ is $\approx 15 \mathrm{meV}^{41,42}$, we conclude that the integration range for our neutron totalscattering measurements extends at least past this energy, and thus the low-energy atomic vibrations are captured in the data.

Analyses of refined atomic configurations. The $3 \mathrm{D}$ local cation-order metric used here to describe a distribution of $\mathrm{Mg}$ and $\mathrm{Nb}$ over the octahedral sites was defined by adopting an approach proposed in ref. ${ }^{16}$ to characterize the $\mathrm{Mg} / \mathrm{Nb}$ ordering from atomic-resolution STEM images of PMN. In this work, the order metric has been equated with the coefficient of variation of intensities for the $\mathrm{Mg} /$ $\mathrm{Nb}$ columns surrounding a $\mathrm{Pb}$ column. For a disordered case, all the $\mathrm{Mg} / \mathrm{Nb}$ columns have similar average atomic numbers/intensities, and the coefficient of variation is zero. In contrast, strong ordering yields large values of this metric. In this case, we defined the ordering metric, $\eta$, as $\eta=\left(n_{1}-n_{2}\right)^{2}+\left(n_{4}-n_{3}\right)^{2}$, where $n_{\mathrm{i}}$ is the fraction of, say, $\mathrm{Nb}$ atoms found in the $i^{\text {th }}$ coordination shell around a given $\mathrm{B}$-cation $(\mathrm{Nb}$ or $\mathrm{Mg}$ ) normalized to the fraction expected for this shell from the average concentration of $\mathrm{Nb}$. The metric was calculated for each B-cation in the configuration. With this definition, clusters of the B-cations with large values of $\eta$ represent chemically ordered regions.

Inverse Fourier-transform filtering of the complex diffuse-scattering amplitude calculated from the refined atomic coordinates was performed by placing spherical masks with diameter $0.2 \AA^{-1}$ around the features of interest. The amplitude of this transform was calculated around each $\mathrm{Pb}$ atom on a grid with a mesh size of $0.05 \AA$. The atoms that provide the largest contribution to the diffuse scattering features used in the inverse Fourier transform are associated with the largest difference between the maximum and minimum amplitudes. The distance vector that corresponds to the spatial locations of these extrema reflects a component of the atomic displacement that participates in the correlations yielding the diffuse feature of interest.
Distortions of the oxygen octahedral framework from an ideal configuration were characterized by introducing a set of deformation modes for the oxygen octahedra. For a more intuitive interpretation, the modes were selected to mimic both typical lattice distortions (e.g., tetragonal, orthorhombic) and vibrational modes (rotations, bending) while providing a complete orthonormal basis. The resulting modes are depicted in Supplementary Fig. 15, whereas their corresponding coordinate-transformation matrices for cubic symmetry are summarized in Supplementary Table 1. All the modes, except for the tetragonal, preserve an octahedron's volume. The orthorhombic and rotational modes describe oxygen coordinates adequately within the limits of the small-angle (linear) approximation.

\section{Data availability}

The experimental data that support the findings of this study are available from the corresponding author upon reasonable request. A file with a representative refined atomic configuration is provided as a part of Supplementary Information, https://doi.org/ 10.6084/m9.figshare.7870955.

\section{Code availability}

The RMCProfile software (executables) can be downloaded from www.rmcprofile.org.

Received: 14 November 2018 Accepted: 15 May 2019

Published online: 21 June 2019

\section{References}

1. Billinge, S. J. \& Levin, I. The problem with determining atomic structure at the nanoscale. Science 316, 561-565 (2007).

2. Keen, D. A. \& Goodwin, A. L. The crystallography of correlated disorder. Nature 521, 303-309 (2015).

3. Smolenskii, G. A. Physical phenomena in ferroelectrics with diffuse phase transition. J. Phys. Soc. Jpn. 28, 26-37 (1970).

4. Cross, L. E. Relaxor ferroelectrics. Ferroelectrics 76, 241-267 (1987).

5. Cohen, R. E. Relaxors go critical. Nature 441, 941-942 (2006).

6. Cowley, R. A., Gvasaliya, S. N., Lushnikov, S. G., Roessli, B. \& Rotaru, G. M Relaxing with relaxors: a review of relaxor ferroelectrics. Adv. Phys. 60 , 229-327 (2011).

7. Burns, G. \& Dacol, F. H. Glassy polarization behavior in ferroelectric compounds $\mathrm{Pb}\left(\mathrm{Mg}_{13} \mathrm{Nb}_{23}\right) \mathrm{O}_{3}$ and $\mathrm{Pb}\left(\mathrm{Zn}_{13} \mathrm{Nb}_{23}\right) \mathrm{O}_{3}$. Solid State Commun. 48, 853-856 (1983).

8. Hiraka, H., Lee, S.-H., Gehring, P. M., Xu, G. \& Shirane, G. Cold neutron study of the diffuse scattering and phonon excitations in the relaxor $\mathrm{Pb}\left(\mathrm{Mg}_{1 / 3} \mathrm{Nb}_{2 / 3}\right) \mathrm{O}_{3}$. Phys. Rev. B 70, 184105 (2004).

9. Jeong, I.-K. et al. Observation of formation of polar nanoregions in $\mathrm{Pb}\left(\mathrm{Mg}_{1 / 3} \mathrm{Nb}_{2 / 3}\right) \mathrm{O}_{3}$ using neutron pair distribution function analysis. Phys. Rev. Lett. 94, 147602 (2005).

10. Naberezhnov, A., Vakhrushev, S., Dorner, B., Straunch, D. \& Moudden, H. Inelastic neutron scattering study of the relaxor ferroelectric $\mathrm{PbMg}_{1 / 3} \mathrm{Nb}_{2 / 3} \mathrm{O}_{3}$ at high temperatures. Eur. Phys. J. B 11, 13-20 (1999).

11. Vakhrushev, S., Ivanov, A. \& Kulda, J. Diffuse neutron scattering in relaxor ferroelectric $\mathrm{PbMg}_{1 / 3} \mathrm{Nb}_{2 / 3} \mathrm{O}_{3}$. Phys. Chem. Chem. Phys. 7, 2340-2345 (2005).

12. Matsuura, M. et al. Composition dependence of the diffuse scattering in the relaxor ferroelectric compound $(1-\mathrm{x}) \mathrm{Pb}\left(\mathrm{Mg}_{1 / 3} \mathrm{Nb}_{2 / 3}\right) \mathrm{O}_{3}-\mathrm{xPTiO}_{3}(0 \leq \mathrm{x} \leq 0.40)$. Phys. Rev. B 74, 144107 (2006)

13. Gehring, P. M. et al. Reassessment of the Burns temperature and its relationship to the diffuse scattering, lattice dynamics, and thermal expansion in relaxor $\mathrm{Pb}\left(\mathrm{Mg}_{1 / 3} \mathrm{Nb}_{2 / 3}\right) \mathrm{O}_{3}$. Phys. Rev. B 79, 224109 (2009).

14. Ganesh, P. et al. Origin of diffuse scattering in relaxor ferroelectrics. Phys. Rev. B 81, 144102 (2010).

15. Bosak, A., Chernyshov, D., Vakhrushev, S. \& Krisch, M. Diffuse scattering in relaxor ferroelectrics: true three-dimensional mapping, experimental artefacts, and modeling. Acta Cryst. A 68, 117-123 (2012).

16. Pasciak, M., Welberry, T. R., Kulda, J., Kempa, M. \& Hlinka, J. Polar nanoregions and diffuse scattering in the relaxor ferroelectric $\mathrm{PbMg}_{1 / 3} \mathrm{Nb}_{2}$ ${ }_{3} \mathrm{O}_{3}$. Phys. Rev. B 85, 224109 (2012).

17. Takenaka, H., Grinberg, I., Liu, S. \& Rappe, A. M. Slush-like polar structures in single-crystal relaxors. Nature 546, 391-395 (2017).

18. Krogstad, M. J. et al. The relation of local order to material properties in relaxor ferroelectrics. Nat. Mater. 17, 718-724 (2018).

19. Glazounov, A. E., Tagantsev, A. K. \& Bell, A. J. Evidence for domain-type dynamics in the ergodic phase of the $\mathrm{PbMg}_{1 / 3} \mathrm{Nb}_{2 / 3} \mathrm{O}_{3}$ relaxor ferroelectric. Phys. Rev. B 53, 11281 (1996).

20. Tagantsev, A. K. \& Glazounov, A. E. Mechanism of polarization response in the ergodic phase of a relaxor ferroelectric. Phys. Rev. B 57, 18-21 (1998). 
21. $\mathrm{Fu}, \mathrm{D}$. et al. Relaxor $\mathrm{Pb}\left(\mathrm{Mg}_{1 / 3} \mathrm{Nb}_{2 / 3}\right) \mathrm{O}_{3}$ : a ferroelectric with multiple inhomogeneities. Phys. Rev. Lett. 103, 207601 (2009).

22. Manley, M. E. et al. Phonon localization drives polar nanoregions in a relaxor ferroelectrics. Nat. Commun. 5, 3683 (2014).

23. Tinte, S., Burton, B. P., Cockayne, E. \& Waghmare, U. V. Origin of the relaxor state in $\mathrm{Pb}\left(\mathrm{B}_{\mathrm{x}} \mathrm{B}_{1-\mathrm{x}}\right) \mathrm{O}_{3}$ perovskites. Phys. Rev. Lett. 97, 137601 (2006).

24. Tucker, M. G., Keen, D. A., Dove, M. T., Goodwin, A. L. \& Hui, Q. RMCProfile: reverse Monte Carlo for polycrystalline materials. J. Phys. Cond. Matter 19, 335218 (2007).

25. Eremenko, M., Krayzman, V., Gagin, A. \& Levin, I. Advancing reverse Monte Carlo structure refinements to the nanoscale. J. Appl. Cryst. 50, 1561-1570 (2017).

26. Al-Barakaty, A., Prosandeev, S., Wang, D., Dkhil, B. \& Bellaiche, L. Finitetemperature properties of the relaxor $\mathrm{PbMg}_{1 / 3} \mathrm{Nb}_{2 / 3} \mathrm{O}_{3}$ from atomistic simulations. Phys. Rev. B 91, 214117 (2015).

27. Cabral, M. J., Zhang, S., Dickey, E. C. \& LeBeau, J. M. Direct observation of local chemistry and local cation displacements in the relaxor ferroelectric PMN-PT. Microsc. Microanal. 22, 1402-1403 (2016).

28. Cabral, M. J., Zhang, S., Dickey, E. C. \& LeBeau, J. M. Gradient chemical order in the relaxor $\mathrm{Pb}\left(\mathrm{Mg}_{1 / 3} \mathrm{Nb}_{2 / 3}\right) \mathrm{O}_{3}$. Appl. Phys. Lett. 112, 082901 (2018).

29. Kopecky, M., Cub, J., Fabry, J. \& Hlinka, J. Nanometer-range atomic order directly recovered from resonant diffuse scattering. Phys. Rev. B 93, 054202 (2016).

30. Warren, B. E. X-Ray Diffraction. (Dover Publications, New York, 1990).

31. Shannon, R. D. Revised effective ionic radii and systematic studies of interatomic distances in halides and chalcogenides. Acta Cryst. A 32, 751-767 (1976).

32. Burton, B. P. \& Cockayne, E. Why $\mathrm{Pb}\left(\mathrm{B}, \mathrm{B}^{\prime}\right) \mathrm{O}_{3}$ perovskites disorder at lower temperatures than $\mathrm{Ba}\left(\mathrm{B}, \mathrm{B}^{\prime}\right) \mathrm{O}_{3}$ perovskites. Phys. Rev. B 60, R12542-R12545 (1999).

33. McInnes, L., Healy, J. \& Astels, S. Hdbscan: hierarchical density based clustering. J. Open Source Softw. 2, 205 (2017).

34. Tkachuk, A. \& Chen, H. Anti-ferrodistortive nanodomains in PMN relaxor. AIP Conf. Proc. 677, 55-64 (2003).

35. Hehlen, B., Al-Sabbagh, M., Al-Zein, A. \& Hlinka, J. Relaxor ferroelectrics: back to the single-mode picture. Phys. Rev. Lett. 117, 155501 (2016).

36. Swartz, S. L. \& Shrout, T. R. Fabrication of perovskite lead magnesium niobate. Mat. Res. Bull. 17, 1245-1250 (1982).

37. Ravel, B. \& Newville, M. ATHENA, ARTEMIS, HEPAESTUS: data analysis for X-ray absorption spectroscopy using IFEFFIT. J. Synch. Rad. 12, 537-541 (2005).

38. Ankudinov, A. L., Ravel, B., Rehr, J. J. \& Conradson, S. D. Real-space multiplescattering calculation and interpretation of X-ray absorption near-edge structure. Phys. Rev. B 58, 7565-7576 (1998).

39. Juhas, P., Davis, T., Farrow, C. L. \& Billinge, S. J. L. PDFGetX3: a rapid and highly automatable program for processing powder diffraction data into total scattering pair distribution functions. J. Appl. Cryst. 46, 560-566 (2013).

40. Butler, B. D. \& Welberry, T. R. Calculation of diffuse scattering from simulated disordered crystals: a comparison with optical transforms. J. Appl. Cryst. 25, 391-399 (1992).

41. Egami, T. \& Billinge, S. J. L. Underneath the Bragg Peaks: Structural Analysis of Complex Materials. (Pergamon Press, Elsevier Ltd, Oxford, 2003).

42. Dmowski, W. et al. Local lattice dynamics and the origin of the relaxor ferroelectric behavior. Phys. Rev. Lett. 100, 137602 (2008).

\section{Acknowledgements}

The ceramic PMN sample used in this study was prepared by Russell Maier. The octahedral deformation-mode analysis was developed by Anton Gagin. We thank the STFC (UK) for access to the ISIS neutron facility. Portions of this research were carried out at the National Synchrotron Light Source II (NIST beamline 6-BM) and Advanced Photon Source (beamline 11-ID-B), the U.S. Department of Energy (DOE) Office of Science User Facilities, operated for the DOE Office of Science by Brookhaven National Laboratory and Argonne National Laboratory, respectively, under Contracts Nos DE-SC0012704 and DE-AC02-06CH11357, respectively.

\section{Author contributions}

M.E. developed the computer algorithms and software, with major contributions from V.K., and performed structural refinements and data analysis. H.Y.P., K.C., J.W., and B.R. collected neutron total scattering, X-ray total scattering, and EXAFS data, respectively. A.B. collected single-crystal diffuse-scattering data and reconstructed the 3D diffuse-intensity distributions. I.L. conceived and guided the study, collected and analyzed data, and wrote the paper with input from M.E. and V.K. All the authors read and approved the paper.

\section{Additional information}

Supplementary Information accompanies this paper at https://doi.org/10.1038/s41467 019-10665-4.

Competing interests: The authors declare no competing interests.

Reprints and permission information is available online at http://npg.nature.com/ reprintsandpermissions/

Peer review information: Nature Communications thanks Jiri Kulda, Hiroyuki Takenaka, Andrew Goodwin and other anonymous reviewers for their contribution to the peer review of this work.

Publisher's note: Springer Nature remains neutral with regard to jurisdictional claims in published maps and institutional affiliations.

Open Access This article is licensed under a Creative Commons Attribution 4.0 International License, which permits use, sharing, adaptation, distribution and reproduction in any medium or format, as long as you give appropriate credit to the original author(s) and the source, provide a link to the Creative Commons license, and indicate if changes were made. The images or other third party material in this article are included in the article's Creative Commons license, unless indicated otherwise in a credit line to the material. If material is not included in the article's Creative Commons license and your intended use is not permitted by statutory regulation or exceeds the permitted use, you will need to obtain permission directly from the copyright holder. To view a copy of this license, visit http://creativecommons.org/ licenses/by/4.0/.

This is a U.S. Government work and not under copyright protection in the US; foreign copyright protection may apply 2019 